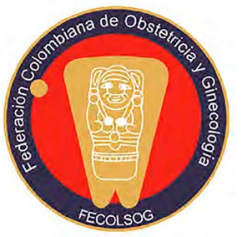

\title{
Deficiencia de yodo en mujeres gestantes indígenas en Colombia: un problema de equidad y de salud pública
}

Javier Eslava-Schmalbach, M.D., M.Sc., Ph.D. ${ }^{1}$; Laura Eslava-González, B. Sc. ${ }^{2}$

a deficiencia de yodo, el bocio, el hipotiroidismo y el hipotiroidismo congénito han sido problemas de salud pública desde hace muchos años, cuya solución ha generado intervenciones comunitarias e individuales a nivel global y local. En Colombia, históricamente se presentaron altas frecuencias de bocio endémico. Rueda-Williamson informa, en escolares de Caldas, una prevalencia de bocio endémico del 83,1\% en 1945, y del 33,9\%, en 1952 (1). Posteriormente, este mismo autor informa que a partir de estrategias como la yodización de la sal en este departamento - que fue uno de los sitios donde primero se introdujo, de manera experimental, la yodización con el fin de prevenir el bocio endémico-, que llevó a cabo el Instituto Nacional de Nutrición, creado en 1963, se disminuyó la frecuencia de bocio en esta población escolar hasta el 1,8\% en 1965 (2). Sin embargo, para 1984, 21 años después de la implementación formal de la yodización en Colombia, se reportaba una frecuencia de bocio endémico del 52\% en la población de Chámeza y de 13\% en Yopal, ambos municipios del departamento del Casanare, con valores promedio de TSH altos en ambas poblaciones (3) . Posteriormente, en el año 2000, se implementa el programa de tamizaje neonatal del hipotiroidismo congénito (4). En 2015 se publica la Encuesta Nacional de la Situación Nutricional (ENSIN), que mostró una deficiencia de yodo del $16,6 \%$ en los menores

1 Líder Grupo de Equidad en Salud, Facultad de Medicina, Universidad Nacional de Colombia; Hospital Universitario Nacional de Colombia, Bogotá (Colombia). 2 Behavioral Neurosciences, Simon Fraser University, Burnaby (Canadá). de 1 a 4 años de etnia indígena, más del doble que la deficiencia para los menores de la misma edad sin pertenencia étnica, en quienes se informó una prevalencia de deficiencia de yodo del 7,5\% (tabla 1) (5). Estas cifras sugieren que en muchas regiones del país esta deficiencia nutricional sigue siendo un problema de salud pública.

En este número de la Revista Colombiana de Obstetricia y Ginecología se presenta el estudio de HerreraMurgueitio et al., realizado en 2019, para identificar la prevalencia de yodo-deficiencia en gestantes indígenas en cinco departamentos de Colombia: Amazonas, Cauca, Córdoba, La Guajira y Meta. Los autores, a partir de una muestra aleatoria de un universo de 5.925 gestantes, encontraron una prevalencia de 33,2 \% de yodo-deficiencia. En este mismo grupo de gestantes, la desnutrición crónica fue del 27,9\% (minmax: 16,6-36,3), la mediana de la línea de pobreza fue de 34,8\% (min-max: 25,4-53,0), la mediana de pobreza extrema de 11,1\% (min-max: 9,1-26,7) y la mediana del analfabetismo de 21,6\% (min-max: 17,2-40,9). La prevalencia de bocio fue de 34,4\%.

Es claro el impacto que la yodo-deficiencia tiene para la salud de las gestantes y, especialmente, la de sus hijos, ya que es causa de anormalidades en el desarrollo neurológico del feto que pueden terminar en dificultades cognitivas, psicomotoras, déficits de percepción y sordera del infante (6-8). Estudios con animales de laboratorio (ratas Wistar) han sugerido cambios moleculares evidentes en el hipocampo de crías nacidas de madres con deficiencia de yodo 
durante el embarazo. Las crías tuvieron una reducción de factores importantes para la reconstrucción y regulación de sinapsis, diferenciación de células, y consolidación y mantenimiento de la memoria en el hipocampo. Estos cambios resultaron en peor rendimiento en pruebas de memoria espacial, como el laberinto acuático de Morris, lo que demuestra los cambios permanentes en la memoria y el aprendizaje en la cría (6). Se ha descrito que el hipocampo es especialmente importante para la consolidación de la memoria a largo plazo y para el aprendizaje (7).

Los efectos neurológicos permanentes que han sido observados en niños cuyas madres tuvieron deficiencia de yodo durante el embarazo dependen de la gravedad de dicha deficiencia. Se ha sugerido que deficiencias más severas pueden llevar a cambios más marcados, incluyendo el cretinismo, enfermedad caracterizada por hipotiroidismo congénito, retardo mental, sordera, dificultades para hablar y déficits psicomotores $(8,9)$. Se ha sugerido, también, que deficiencias más severas durante el embarazo causan menores puntajes en las pruebas de coeficiente intelectual en los niños. Deficiencias moderadas o leves pueden llevar a déficits de percepción y cognición en niños, e incluso al desarrollo de trastorno por déficit de atención con hiperactividad (8). Por el contrario, el tratamiento de estas deficiencias durante el primer trimestre del embarazo puede mejorar el desarrollo neurológico y llegar a prevenir consecuencias neurológicas permanentes (8-10).

Como destacan Herrera-Murgueitio et al., es la primera vez que se explora la deficiencia de yodo y la presencia de bocio en gestantes indígenas en Colombia, población en donde se han descrito importantes inequidades asociadas a la mortalidad materna. En dos estudios previos se había mostrado que las brechas de mortalidad materna en el país se encontraban principalmente en los departamentos más pobres (11) o con necesidades básicas insatisfechas más profundas (12). En algunos de estos departamentos residen las mujeres gestantes indígenas descritas en el estudio.

De la misma manera, la ENSIN-2015 muestra profundas diferencias en los determinantes sociales de la salud en su relación con la etnia indígena, y refleja que la yodo-deficiencia es una variable proxy de una problemática estructural que margina a estas poblaciones de los potenciales desarrollos que se dan en las zonas urbanas o en las grandes ciudades. Esta marginación, de acuerdo con el nivel de su yodo-deficiencia, ubica a las gestantes del estudio en una situación similar a la del año 1952, cuando las investigaciones experimentales en Colombia (2) lograron bajar el bocio endémico a niveles parecidos a los que las gestantes indígenas tenían en el estudio de Herrera-Murgueitio et al. para 2019. Otros autores muestran una muy estrecha relación entre la deficiencia de yodo de las madres con la de sus hijos, por lo que ellos están ubicados en el mismo entorno con las mismas deficiencias estructurales y de inseguridad alimentaria (13).

A partir de la ENSIN-2015 se hacen evidentes en la población indígena los problemas de vivienda, acceso a acueducto público, la baja educación de las madres de menores de 5 años, la informalidad en el empleo, la pobreza, la alta inseguridad alimentaria y la desnutrición global, cuando se compara con el grupo sin pertenencia étnica (tabla 1).

En un trabajo previo sobre los determinantes sociales de la salud asociados a la muerte prematura por desnutrición en menores de 5 años (14) se propone un modelo explicativo de dicha mortalidad que incluye: 1) las condiciones socioeconómicas y políticas; 2) el entorno, con las condiciones ambientales, la ruralidad, el conflicto armado, la disponibilidad de alimentos, el agua segura y el acceso a servicios públicos; 3) los padres y cuidadores, en donde se considera la pertenencia étnica, la educación, las condiciones laborales y de vivienda; 4) los aspectos de tipo individual. Muchas de estas variables se muestran en la tabla 1 y reflejan el nivel de vulnerabilidad que tiene la población indígena en Colombia, no solo para hipotiroidismo congénito, sino para mortalidad prematura por desnutrición.

Por su parte, una exploración realizada por el Institute for Health Metrics and Evaluation (IHME) 


\begin{tabular}{|c|c|c|}
\hline Determinante evaluado & $\begin{array}{l}\text { Indígena } \\
\text { (\%) (IC } 95 \%)\end{array}$ & $\begin{array}{l}\text { Sin pertenencia étnica } \\
(\%)(\text { IC } 95 \%)\end{array}$ \\
\hline Déficit cuantitativo de vivienda & $* 10,9(8,1-14,6)$ & $6,5(5,8-7,2)$ \\
\hline Déficit cualitativo de vivienda & $51,2(43,7-58,6)$ & $18,7(17,4-20)$ \\
\hline Con acueducto público & $42,2(34,5-50,3)$ & $79,8(78,3-81,2)$ \\
\hline $\begin{array}{l}\text { Madres de menores de } 5 \text { años, con educación secundaria completa y } \\
\text { superior incompleta }\end{array}$ & $25(18,8-32,5)$ & $54,7(52,5-56,8)$ \\
\hline Informalidad en el empleo & $* 81(74,1-86,5)$ & $43,6(41,7-45,4)$ \\
\hline Hogares en el cuartil más bajo del índice de riqueza & $67,8(61,7-73,3)$ & $22,5(20,9-24,2)$ \\
\hline Hogares con inseguridad alimentaria & $76,9(74,5-79,1)$ & $52,3(54,2-55,9)$ \\
\hline Autoconsumo & $50,2(43,9-56,5)$ & $14,9(13,9-16,0)$ \\
\hline Estrategias de afrontamiento en últimos 7 días por inseguridad alimentaria & $71,3(66,2-75,9)$ & $49,9(48,7-51,1)$ \\
\hline $\begin{array}{l}\text { Hogares con menores de } 18 \text { años que enviaron niño o niña con vecinos o } \\
\text { familiares por falta de alimento }\end{array}$ & $15(12,0-18,6)$ & $7,7(7,2-8,2)$ \\
\hline Dieta mínima aceptable en menores de 2 años & $24,7(18,6-32,1)$ & $37,4(34,6-40,3)$ \\
\hline Deficiencia de yodo en niños de 1 a 4 años & $16,6(12,3-21,9)$ & $7,5(6,4-8,7)$ \\
\hline Desnutrición global en niños de 0 a 4 años & $7,2(5,1-10,2)$ & $3,0(2,4-3,7)$ \\
\hline Mujeres gestantes del total, de 10 a 19 años & $24,8(16,1-36,2)$ & $19,2(16,2-22,5)$ \\
\hline
\end{tabular}

Fuente: elaboración propia a partir de datos tomados de ENSIN-2015 (5).

* Coeficiente de variación 15 a 20 \%, precisión aceptable, según ENSIN-2015.

muestra los años de vida ajustados por discapacidad en Colombia (Disability Adjusted Life Years - DALY) por desórdenes asociados a la deficiencia de yodo; al respecto, aunque Colombia tuvo un descenso en los DALY, entre 2005 y 2010, sus valores volvieron a ser similares a aquellos que tenía en 1990 (15). En otras palabras, un retroceso de 30 años.

Además de las políticas de yodización de la sal y del tamizaje del hipotiroidismo congénito, Colombia lanzó una estrategia para la prevención y el control de las deficiencias de micronutrientes (16) que incluye el yodo, pero que al parecer, por los resultados del estudio de Herrera-Murgueitio et al., no ha alcanzado a llegar aún a las regiones donde se encuentra la población indígena, o tuvo poca sostenibilidad, como ocurrió con la declaración de la eliminación de los desórdenes por deficiencia de yodo en Colombia, en 1998 (17).
Los resultados de Herrera-Murgueitio et al. podrían extrapolarse no solo a las 5.925 gestantes indígenas que hacen parte del universo del estudio (al menos 2.000 hogares en situación de inseguridad alimentaria y nutricional), sino a otras poblaciones que también se encuentran marginadas al interior de las ciudades o de las zonas rurales y que, con la misma afectación de los determinantes sociales, pasan por problemas similares de inseguridad alimentaria durante sus vidas y sus embarazos. El hipotiroidismo congénito es una condición prevenible, evitable y, a toda vista intolerable, por sus potenciales consecuencias (18).

La política de yodización de sal requiere una revisión profunda, así como también el impacto que tiene en quienes la consumen y en quienes no tienen acceso a ella. El monitoreo de los niveles de yodo en orina, de la ingesta de yodo, de tiroglobulina (19) o de otras 
formas de evaluarlo (20) debe hacer parte del control prenatal usual de la paciente gestante, de los niños en crecimiento y de la población en general. De todas formas, sus resultados serán también un reflejo de los determinantes sociales de la salud que le subyacen.

\section{REFERENCIAS}

1. Rueda-Williamson R, Pardo Téllez F, Piedrahíta Hoyos F, Ariza Macías J, Uribe Naranjo L. La efectividad de la yodación de la sal en la prevención del bocio endémico en Colombia: resultados en escolares de Caldas. Rev Soc Colomb Endocrinol. 1966;4(2):271-6.

2. Rueda-Willianson R, Pardo Téllez F. La prevención del bocio endémico en Colombia. Bol Sanit Panam. 1966:495-503.

3. Carrillo J, Mockus I, Casadiego C, Salcedo L, Tarazona B, Galarza L, et al. Evaluación de desórdenes por deficiencia de Yodo (DDI) en dos poblaciones de Casanare, Colombia, 1984-1986. Temas de Pediatría. Vevey: Nestlé, Servicio de Información Científica; 1986

4. Instituto Nacional de Salud. Manual de tamizaje neonatal por el laboratorio. Bogotá: Instituto Nacional de Salud; 2014. 80 p.

5. Gobierno de Colombia. Encuesta Nacional de Situación Nutricional ENSIN 2015. Instituto Colombiano de Bienestar Familiar, Instituto Nacional de Salud, Universidad Nacional de Colombia; 2015.

6. Liu Y, Zhang L, Li J, Shan Z, Teng W. Maternal marginal iodine deficiency affects the expression of relative proteins during brain development in rat offspring. J Endocrinol. 2013;217(1):21-9. https:// doi.org/10.1530/JOE-12-0410

7. Gerges NZ, Alkadhi KA. Hypothyriodism impairs late LTP in CA1 region but not in dentate gyrus of the intact rat hippocampus: MAPK involvement. Hippocampus. 2004;14: 40-45. https://doi.org/10.1002/hipo.10165

8. Caron P. Neurocognitive outcomes of children secondary to mild iodine deficiency in pregnant women. Ann Endocrinol. 2015;76(3):248-52. https:// doi.org/10.1016/j.ando.2015.01.001

9. Zimmermann MB. The role of iodine in human growth and development. Semin Cell Dev Biol.
2011;22(6):645-52. https://doi.org/10.1016/j. semcdb.2011.07.009

10. Lavado-Autric R, Ausó E, García-Velasco JV, Arufe MC, Escobar del Rey F, Berbel P, et al. Early maternal hypothyroxinemia alters histogenesis and cerebral cortex cytoarchitecture of the progeny. J Clin Invest. 2003;111(7):1073-82. https://doi.org/10.1172/ JCI16262

11. Cárdenas-Cárdenas LM, Cotes-Cantillo K, ChaparroNarváez PE, Fernández-Niño JA, Paternina-Caicedo A, Castañeda-Orjuela C, et al. Maternal mortality in Colombia in 2011: A two level ecological study. PLoS One. 2015;10(3):1-14. https://doi.org/10.1371/ journal.pone.011894

12. Sandoval-Vargas Y, Eslava-Schmalbach J. Inequidades en mortalidad materna por departamentos en Colombia para los años 2000-2001, 2005-2006 y 2008-2009. Rev Salud Pública. 2013;15(4):577-89.

13. Wong EM, Sullivan KM, Perrine CG, Rogers LM, Peña-Rosas JP. Comparison of median urinary iodine concentration as an indicator of iodine status among pregnant women, school-age children, and nonpregnant women. Food Nutr Bull. 2011;32(3):206-12. https:// doi.org/10.1177/156482651103200304

14. Agudelo Ibáñez DR. Mortalidad por desnutrición infantil en menores de 5 años y sus determinantes a nivel municipal en Colombia 1998-2016. Bogotá: Universidad Nacional de Colombia; 2019.

15. Institute for Health Metrics and Evaluation. GBD Compare | IHME Viz Hub. Global Burden of Disease. 2017. Disponible en: https://vizhub.healthdata.org/ gbd-compare/

16. Ministerio de Salud y Protección Social. Estrategia Nacional para la prevención y control de las deficiencias de micronutrientes en Colombia 2014-2021. Bogotá: Ministerio de Salud y Protección Social; 2015. 72 p.

17. Ministerio de Salud y Protección Social. Definición del problema para el análisis del impacto normativo relacionado con sal para consumo humano. Bogotá: Ministerio de Salud y Protección Social; 2019.

18. Rahmani K, Yarahmadi S, Etemad K, Koosha A, Mehrabi Y, Aghang N, et al. Congenital hypothyroidism: 
Optimal initial dosage and time of initiation of treatment: A systematic review. Int J Endocrinol Metab. 2016;14(3). https://doi.org/10.5812/ijem.36080

19. Katko M, Gazso AA, Hircsu I, Bhattoa HP, Molnar Z, Kovacs B, et al. Thyroglobulin level at week 16 of pregnancy is superior to urinary iodine concentration in revealing preconceptual and first trimester iodine supply. Matern Child Nutr. 2018;14(1). https://doi. org/10.1111/mcn. 12470

20.Zimmermann MB, Andersson M. Assessment of iodine nutrition in populations: Past, present, and future. Nutr Rev. 2012;70(10):553-70. https://doi. org/10.1111/j.1753-4887.2012.00528.x 